\title{
Symmetric supercapacitors based on porous 3D interconnected carbon framework
}

Bello Abdulhakeem*, Barzegar Farshad, Momodu Damilola, Dangbegnon Julien, Taghizadeh Fatemeh, and Manyala Ncholu*

Department of Physics, Institute of Applied Materials, SARChI Chair in Carbon Technology and Materials, University of Pretoria, Pretoria 0028, South Africa.

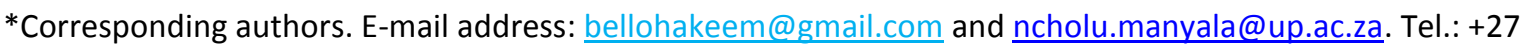
012420 3549; Fax: +270124202516 (N. Manyala)

\section{Abstract}

The construction and design of novel porous carbons for electric double-layer capacitors (EDLCS) application to meet the increasing demand and supply of energy is eminent. This is important because the pore volume (PV)/micropore volume (MV) in the porous network architecture of the carbon is mostly responsible for the ion traps in energy storage. Three dimensional carbon materials based on graphene materials with relatively high specific surface area (SSA) represents a promising material candidate for EDLCs applications. In this work, we synthesized highly porous carbon from graphene foam (GF) and polyvinyl alcohol PVA as a sacrificial template, and investigate their performance as electrodes for EDLCS applications. The as-produced carbons present a fairly large surface area $\left(502 \mathrm{~m}^{2} \mathrm{~g}^{-1}\right)$, and a highly porous interconnected framework with mesopore walls and micropore texture which are suitable as electrode for energy storage. As electrode material in a symmetric configuration the activated graphene foam (AGF) showed a specific capacitance of $65 \mathrm{~F} \mathrm{~g}^{-1}$, energy density of $12 \mathrm{Wh} \mathrm{kg}^{-1}$, power density of $0.4 \mathrm{~kW} \mathrm{~kg}^{-1}$, good rate performance and excellent long term stability in $1 \mathrm{M} \mathrm{Na}_{2} \mathrm{SO}_{4}$ aqueous with no capacitance loss after 3000 cycles. 
Keywords: Graphene foam, polyvinyl alcohol, $\mathrm{KOH}$ activation, symmetric supercapacitor.

\section{$1 \quad$ Introduction}

Electric double-layer capacitors (EDLCs) are charge-storage devices with a high power density, long cyclic life and low maintenance cost that have found applications in portable electronics, memory backup sources, hybrid electric vehicles, regenerative and renewable energy systems [1]. The storage mechanism of EDLCs is based on reversible adsorption of ion across the interface of electrode and electrolyte when a voltage is applied [2]. In practice, most EDLCs make use of carbon due to their low cost, excellent conductivity, chemical stability, and adjustable structural properties and specific surface area (SSA). Other carbon-based materials, metal-oxides, and conducting polymers are also considered due to their pseudocapacitive properties. Commercially, the most commonly used material for EDLCS application is the activated carbon due to its fairly high electrical conductivity, high surface area and are usually operated with organic electrolyte [3]. The use of organic or ionic electrolyte increases the energy density of the EDLC devices because the energy is a function of the voltage squared. It has also been shown that organic electrolyte can operate up to $3.5 \mathrm{~V}$ and ionic liquids up to $5.0 \mathrm{~V}$ [4]. However, there are many disadvantages associated with them: some of which include low ionic conductivity (high internal resistance), highly flammable, high electrolyte leakage and are quite expensive. On the other hand aqueous electrolytes have high ionic conductivity and high mobility of proton transport mechanism which are required to achieve low internal resistance for development of high performance supercapacitor devices. Therefore, most research activities are focussed on the use of aqueous electrolyte for commercial application by trying to extend the potential window of their electrode materials $[5,6]$. 
Recently the developments of high performance EDLCs have been based on porous carbons because of the fact that the porous morphology provides adequate mesopores and micropores, the Brunauer-Emmett-Teller (BET) specific surface area (SSA), pore size distribution (PSD) and other surface functionalities of the porous carbon combined with size of the ion from the electrolyte provide improved performance. Although, It has been shown that a large SSA with adequate distribution of mesopores and micropores is one of the most important parameters that is necessary to understand the performance of porous EDLCS carbon electrode but a definite relationship between the capacitance and SSA has not been clearly shown $[7,8]$. Using porous carbon is favourable because the pore structure governs the measured capacitance, energy storage and power delivery capability of the material. These result from contribution due to the total surface of micropores $\left(S_{\text {micro }}\right)$ and the surface of larger pores (mesopores and macropores) [7]. It is worth stating that the macropores $(>50 \mathrm{~nm})$ makes virtually no contribution to the total capacitance but act as the ion buffering reservoir [9], while micropores ( $<2 \mathrm{~nm}$ in size) serve as the ion traps for energy storage, and mesopores $(2<50 \mathrm{~nm})$ act as the ion transport pathways for power delivery $[9,10]$.

Several synthesis techniques for porous carbons have been explored for different applications [11-14]. Template-based approaches are the most widely used as an efficient technique for production of porous carbon with highly tunable properties such as pore distributions, pore textures, optimized hierarchical structures and surface functionalities $[14,15]$. The main challenge with template synthesis is that it relies on the use of inorganic materials sources such as silica and zeolite, which are expensive and has hindered this technique in large scale production $[16,17]$. An alternative approach to the template 
method in preparing porous carbon materials is the direct use of chemical and physical methods which have emerged as a novel strategy and raised considerable interests amongst researchers because they are well known techniques and allow synthesis of materials with tunable properties $[18,19]$. The chemical treatment usually results in the formation of aerogel or hydrogel materials and the physical treatment (activation) mostly with $\mathrm{KOH}, \mathrm{ZnCl}_{2}$ and $\mathrm{H}_{3} \mathrm{PO}_{4}$ leads to the formation of porous carbons [20-22]. The formation mechanism of porous carbon with $\mathrm{KOH}$ has been attributed to carbon gasification by the removal of $\mathrm{CO}_{2}$ and the oxygen related material contained in the matrix. During the activation process, $\mathrm{CO}_{2}$ formed from $\mathrm{K}_{2} \mathrm{CO}_{3}$ in the heat treatment is released and becomes significant at high temperatures. The released $\mathrm{CO}_{2}$ then react with carbon atoms to open up closed pores and enlarge existing micropores. Meanwhile, the potassium-containing compounds, such as $\mathrm{K}_{2} \mathrm{O}$ and $\mathrm{K}_{2} \mathrm{CO}_{3}$, can be reduced by carbon to form $\mathrm{K}$ metal, thus causing the carbon gasification and hence the formation of pores [22]. The activation process takes places according to the following reaction [22];

$6 \mathrm{KOH}+\mathrm{C} \leftrightarrow 2 \mathrm{~K}+3 \mathrm{H}_{2}+2 \mathrm{~K}_{2} \mathrm{CO}_{3}$

In recent time there have been reports on activation of graphene based materials, for examples Yanwu Zhu et. al. have produced carbon materials assisted by microwaveexpansion called a-MEGO with exceptional properties and demonstrated excellent electrochemical properties in ionic electrolytes [23,24]. Recently Zhang et. al. [25] produced porous 3D graphene-based bulk materials with exceptional supercapacitor performance in ionic liquid with a specific capacitance of $92 \mathrm{~F} / \mathrm{cm}^{3}$ and energy density of $39 \mathrm{Wh} /$ liter in $\mathrm{EMIMBF}_{4}$ electrolyte respectively. However, it is worth stating that most of these activations are based on graphene oxide obtained from the usual Hummer's method and to the best of 
our knowledge there has been no report on the activation of graphene foam derived from the chemical vapor deposition technique (CVD) and this is the first report on a symmetric supercapacitors based on activated graphene foam operating at $1.6 \mathrm{~V}$ in a neutral aqueous electrolyte.

\section{Experimental}

Scheme 1 shows the schematic route used to prepare the porous carbon in this study. Briefly, graphene foam (GF) and polyvinyl alcohol (PVA) were used as starting material for the production of hydrogel, which was then used to produce porous carbon materials after the activation process. Graphene foam (GF) was prepared by chemical vapour deposition (CVD) onto a catalytic nickel foam (Alantum Innovations in Alloy Foam Munich, Germany) as reported in our previous work [26], and $100 \mathrm{mg}$ of the GF was dispersed in $0.1 \mathrm{~g} / \mathrm{ml}$ of (PVA) in a vial glass by ultrasonication followed by addition of $1.5 \mathrm{ml}$ of hydrochloric acid $(\mathrm{HCl})$ to the solution as a cross linker and the resultant mixture was hydrothermally treated in an autoclave heated up to $190{ }^{\circ} \mathrm{C}$ and maintained at this temperature for 12 hours to polymerize the mixture [25]. The resulting hydrogel was washed with deionized water and dried for 6 hours. The as-prepared hydrogel was then soaked in aqueous $\mathrm{KOH}$ solution with a $\mathrm{KOH} /$ hydrogel mass ratio of 7 for 24 hours [24]. The composite mixture was then placed in a horizontal tube furnace ramped from room temperature to $800^{\circ} \mathrm{C}$ at $10{ }^{\circ} \mathrm{C} /$ minute under argon gas flow for 1 hour of activation. This procedure transforms the composite hydrogel into carbon materials consisting of a continuous pore network distribution. The activated material denoted as AGF was neutralized with $0.1 \mathrm{ml} \mathrm{HCl}$, washed with deionised water and dried at $120^{\circ} \mathrm{C}$ for 12 hours after which samples were characterized. 


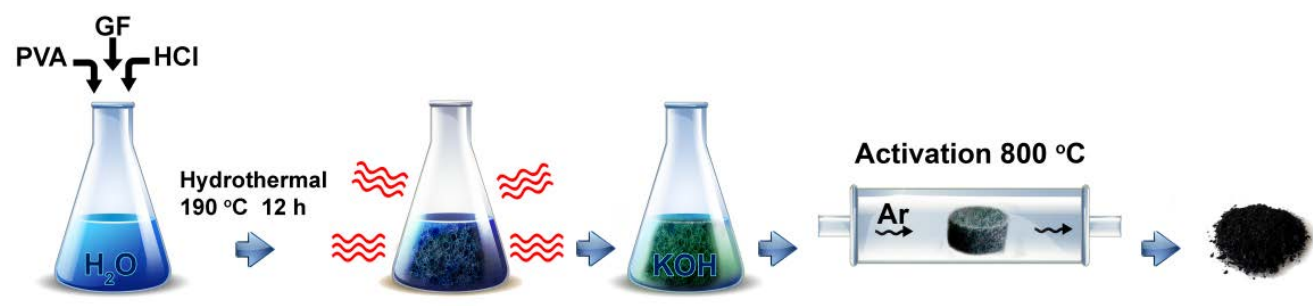

Scheme 1 Preparation of porous carbon from GF and PVA 


\subsection{Material Characterization}

Raman spectroscopy measurements was carried out using a Jobin Yvon Horiba TX 6400 micro-Raman spectrometer equipped with a triple monochromator system to eliminate contributions from Rayleigh lines and using LabSpec (Ver. 5.78.24) analytical software. All the samples were analyzed with a $514 \mathrm{~nm}$ argon excitation laser ( $1.5 \mathrm{~mW}$ laser power on the sample to avoid possible thermal effects and beam damage). The sample was also characterized using powder X-ray diffraction (XRD). An XPERT-PRO diffractometer (PANalytical BV, Netherlands) with theta/theta geometry, operating a cobalt tube at $35 \mathrm{kV}$ and $50 \mathrm{~mA}$, was used. The XRD patterns of all specimens were recorded at room temperature in the $20.0^{\circ}-80.0^{\circ} 2 \theta$ range with a counting time of 15.240 seconds per step. Qualitative phase analysis of the sample was conducted using the X'pert Highscore search match software. Nitrogen sorption isotherms were obtained using Micromeritics TriStar II 3020 (version 2.00), the samples were outgassed for 12 hours at $150{ }^{\circ} \mathrm{C}$. BET surface areas were calculated from the relative pressure range $0.01<\mathrm{P} / \mathrm{P}_{0}<0.2$ of the adsorption branch for the activated carbon. Pore size and pore volume were obtained using Barrett-JoynerHalenda (BJH) method from the desorption branch of the isotherm. Scanning electron microscopy (SEM) was carried out with a Zeiss Ultra Plus 55 field emission scanning electron microscope (FE-SEM) operated at an accelerating voltage of $2.0 \mathrm{kV}$. However, due to the conductivity of the material SEM micrographs were taken without metallic coating. The density of the produced material was determined with a Micrometrics AccuPyc II 1340 V1.03 gas pycnometer operating with helium, using a sample holder which had a maximum volume of $1 \mathrm{~cm}^{3}$. 


\subsection{Coin cell Preparation and Electrochemical Tests}

For the preparation of electrodes, the activated carbon material were mixed with Polyvinylidene difluoride (PVDF) binder with a weight ratio of 9: 1 and was homogenized in an agate mortar, dispersed in 1-methyl-2-pyrrolidinone (NMP) solvent to form a paste. The paste was then coated onto a nickel foam grid as current collector with dimensions of $1 \mathrm{~cm}$ $\mathrm{x} 1 \mathrm{~cm}$ and circular disk with diameter of $1.5 \mathrm{~cm}$ and dried at $60{ }^{\circ} \mathrm{C}$ in an oven for 8 hours to ensure complete evaporation of the NMP and used as electrodes. Typical mass loading of the electrodes was between $5.0-10.0 \mathrm{mg} / \mathrm{cm}^{2}$ in accordance with true performance testing and best-practice method to determine the electrode performance for EDLCs $[27,28]$. Before fabrication of the cell, the two carbon electrodes with similar masses were first prewetted with electrolyte $\left(1 \mathrm{M} \mathrm{Na}_{2} \mathrm{SO}_{4}\right)$, separated with a glass microfiber filter paper as the separator, and then packed into a two-electrode cell.

Electrochemical measurements were performed using a Bio-logic SP-300 potentiostat in both the two and three electrode configuration with AGF as the working electrode, glassy carbon plate as the counter electrode and $\mathrm{Ag} / \mathrm{AgCl}(3 \mathrm{M} \mathrm{KCl})$ serving as the reference electrode. EIS studies were carried out in the frequency range of $100 \mathrm{kHz}-10 \mathrm{mHz}$ with the open circuit potential. Specific capacitance (per unit mass) values were estimated from both CV curves equation 1 and charge-discharge curves using equations 2 [27].

$C_{s p}=2 \int I d t / V m$

where $I$ is the average current (A) measured, $m$ is the mass of the electrode $(\mathrm{g})$ and $V$ is the applied potential window measured in volts (V).

$C_{s p}=2 I \Delta t / m \Delta V$ 
where $i$ is the constant current for charge-discharge, $\Delta t$ is the discharge time measured in seconds (s) and $\Delta V$ is the voltage window, $m$ is the mass of a single electrode in the cell. The mass loading of the activated carbon per electrode was $4 \mathrm{mg}$. The energy and power densities were calculated based on following equations:

$E_{\text {density }}=0.5 C_{s p} V^{2}$

$P_{\text {density }}=E_{\text {density }} / t$

$V$ is maximum cell potential in (V) after the IR drop has been subtracted, $C_{s}$ is the total specific capacitance and $t$ is the discharge time measured in seconds. The Volumetric capacitance was calculated by multiplying the gravimetric capacitance by the density of the material $\left(0.1864 \mathrm{~g} \mathrm{~cm}^{-3}\right)$.

\section{$3 \quad$ Results and Discussion}

\subsection{Structural and morphological characterization}

Raman and XRD play important role in structural characterization of defects in carbon based materials. The characterization of the AGF sample by means of Raman scattering and X-ray diffraction (XRD) is shown in Figure 1 (a) and (b) respectively. Figure 1 (a) shows the 2 prominent $D$ and $G$ peaks, suggesting that the produce AGF is made up of highly disordered graphitic structures created by the formation mechanism of the AGF. The G band at around $1587 \mathrm{~cm}^{-1}$ corresponds to an ideal graphitic lattice vibrational mode with $E_{2 G}$ symmetry and the $D$ band which appears at about $1340 \mathrm{~cm}^{-1}$ corresponds to graphitic lattice vibration mode with $A_{1 g}$ symmetry and known to be characteristics of defect or disorder in the material. The intensity of the G-band to the intensity of the $D$-band $\left(R=I_{D} / I_{G}\right)$ of 1.2 , indicates 

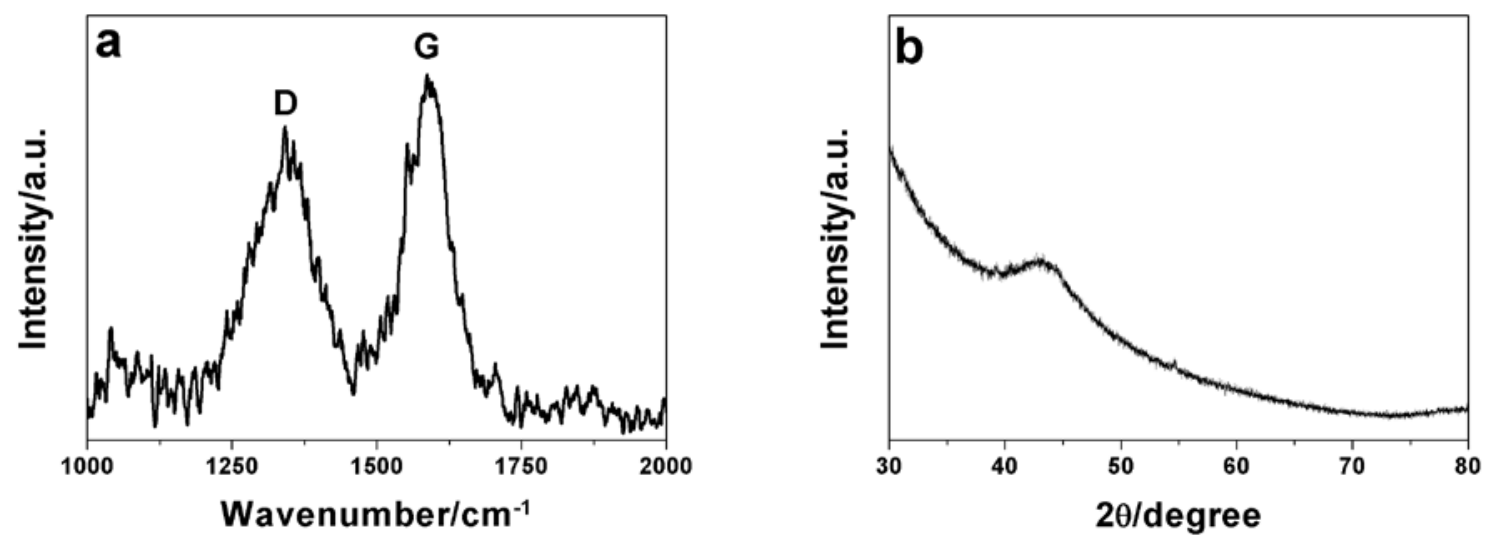

Figure 1 (a) Raman spectrum and (b) Powder X-ray diffraction of the AGF 
a low degree of graphitic crystalline structure [29]. Figure (b) shows the diffraction pattern of the produced AGF and exhibits a weak and broad reflection with intensity maxima at $43.6^{\circ}$ which represent the 101 plane. The broad peak is an indication of the amorphous and disordered state of the AGF which corroborate the highly disordered structure in the sample confirming Raman scattering data.

The morphologies of the hydrogel precursors play a critical role in shaping the structures of the derived carbon products. Figure 2 shows the SEM micrographs of the produced activated carbon. The morphology of the samples contained large density of porous cavities which were formed due to the removal of the impregnated $\mathrm{KOH}$ and $\mathrm{KOH}$ derived compounds during activation.

Figure 2 (a) shows a high magnification image of the activated carbon revealing that the porous cavities are interconnected in size of several micrometres with a substantial amount of macropores which provide sufficient reservoirs for electrolyte which is highly desirable for enhanced supercapacitor electrode performance.

The nitrogen $\left(\mathrm{N}_{2}\right)$ gas sorption experiment was performed to quantify the accessible surface area and pore structure of the activated sample. Figure 3 shows the $\mathrm{N}_{2}$ isotherms and pore size distribution (PSDs) of the as-produced carbons. As observed in Figure 3 (a), the activated samples demonstrate a type $\mathrm{H} 4$ isotherm, which features parallel and almost horizontal curves attributed to the adsorption/ desorption in narrow slit-like pores which is also associated with the capillary condensation in any well-developed mesoporous materials $[30,31]$. At low pressure, the PSDs in Figure 3 (b) with increase in the pore distribution below $3 \mathrm{~nm}$ is an indication of the existence of micropore in this sample and the hysteresis 

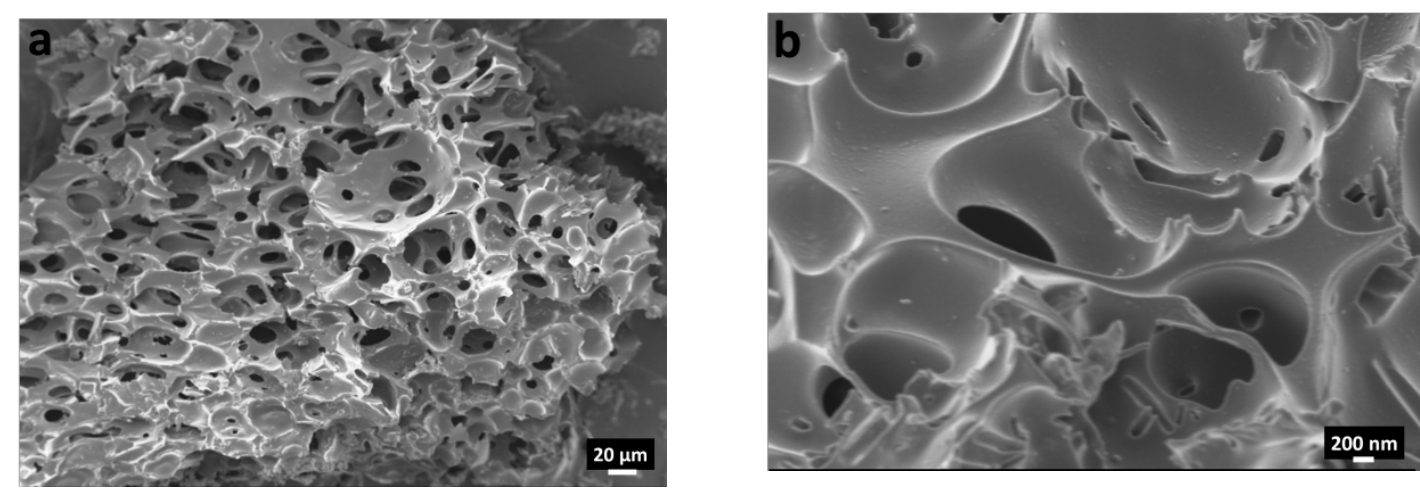

Figure 2 SEM of porous carbon synthesized using the GF and PVA hydrogel 

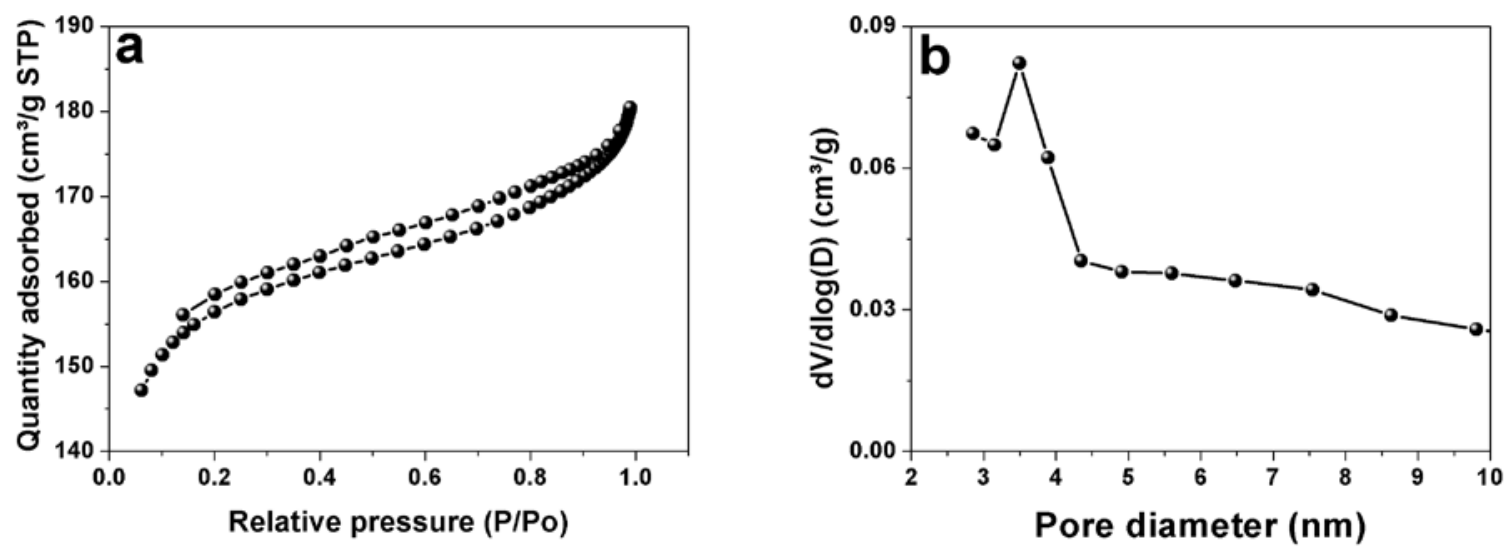

Figure 3 Nitrogen adsorption-desorption isotherm loops and (b) the BJH pore size distribution curves, calculated from desorption branch of isotherm 
loop at the high pressure indicated the mesoporous structure of the sample with a specific surface area of $502 \mathrm{~m}^{2} / \mathrm{g}$ and pore volume of $0.24 \mathrm{~cm}^{3} / \mathrm{g}$.

\subsection{Electrochemical performance}

The electrochemical performance of the electrode in $1 \mathrm{M}$ sodium sulphate $\left(\mathrm{Na}_{2} \mathrm{SO}_{4}\right)$ is presented below. The cyclic voltammograms (CV) of the activated carbon in a three electrode configuration with different operating potential windows are shown in Figure 4 (a) and (b). From this Figure the CV curves of the activated electrode demonstrate the typical rectangular shape for carbon materials at scan rates of $10 \mathrm{mV} / \mathrm{s}$ and $25 \mathrm{mV} / \mathrm{s}$ in both negative ( -0.8 to $0 \mathrm{~V}$ vs. $\mathrm{Ag} / \mathrm{AgCl})$ and positive windows $(-0.3$ to $0.7 \mathrm{~V}$ vs. $\mathrm{Ag} / \mathrm{AgCl})$ with a well-defined EDLC (non-faradaic) behaviour suggesting that the porous electrodes are polarizable.

The CVs of a symmetric AGF//AGF which combines both negative and positive potential window is shown in Figure 4 (c). At a potential window of $1.8 \mathrm{~V}$ a current leap is observed which could be due to the evolution of gases or oxygenated surface functionalities generated from both the positive and/or negative electrode and the reactions with the electrolyte [6,32]. At a potential window of $1.6 \mathrm{~V}$ a well-defined EDLC behaviour which is demonstrated by the typical rectangular shape is observed. The constant current chargedischarge $(C D)$ was also measured at 1.6 and $1.8 \mathrm{~V}$ and is presented in Figure 4 (d). The symmetrical triangular shape of the CD corroborates to the formation of a double layer capacitor. Although the CD curve could reach an upper potential limit of $1.8 \mathrm{~V}$ all measurements performed on the symmetric cell was at $1.6 \mathrm{~V}$ to avoid oxidation of the electrode material. 

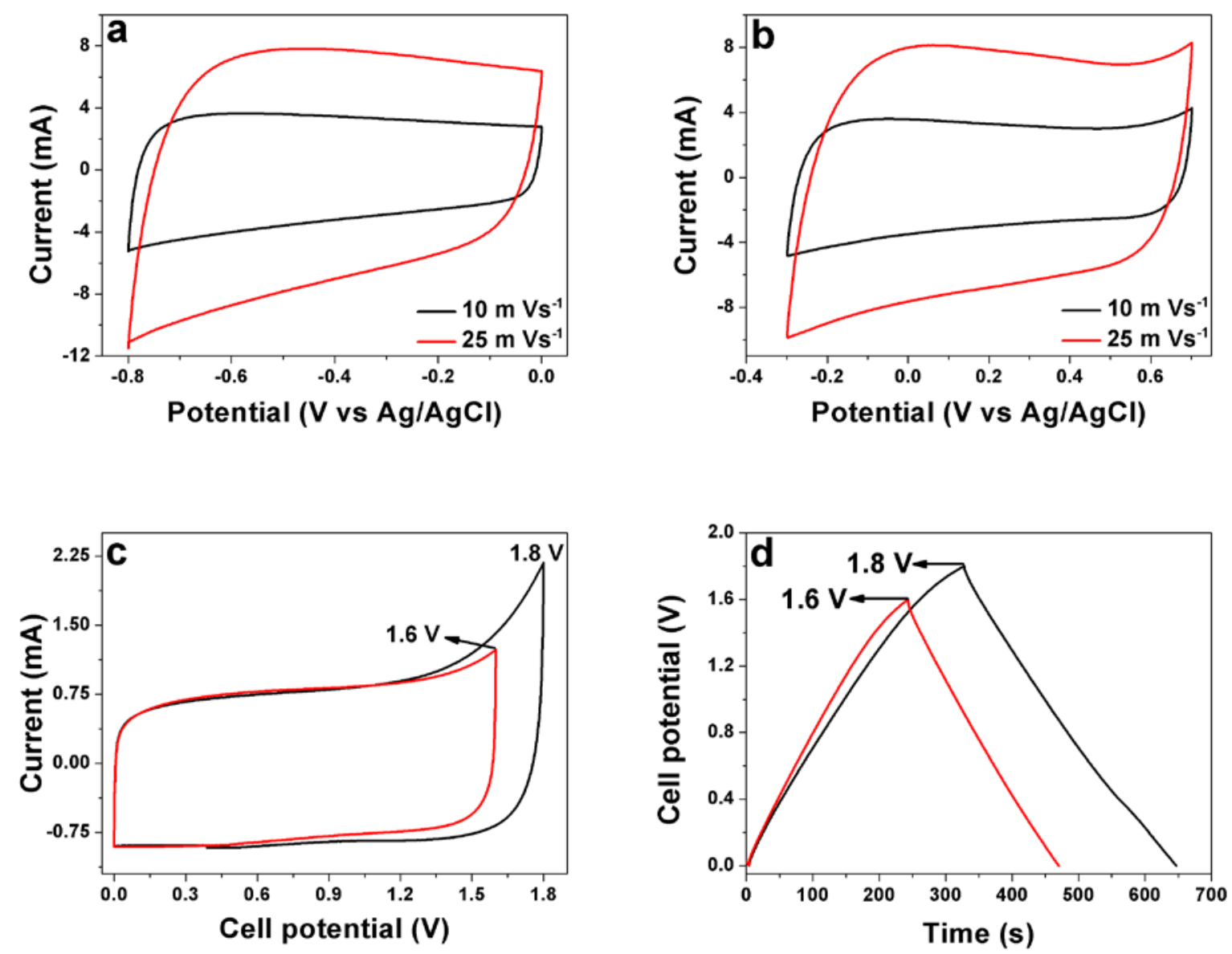

Figure $4 \mathrm{CVs}$ of the AGF electrode in $(\mathrm{a}, \mathrm{b})$ negative and positive voltage windows respectively, (c) CVs of a symmetric AGF/AGF supercapacitor device with different potential windows and (d) constant time charge discharge curves of the AGF device at $1.6 \mathrm{~V}$ and $1.8 \mathrm{~V}$. 
Figure 5 (a) shows CVs of the symmetric AGF//AGF between 0 and $1.6 \mathrm{~V}$ at different scan rates from 5 to $100 \mathrm{mV} \mathrm{s}^{-1}$. Above $20 \mathrm{mV} \mathrm{s}^{-1}$, the cell maintains its rectangular shape which shows that the AGF electrode materials have good rate capability. This is desirable for rapid and efficient charge and discharge of high power devices. However, at scan rate of $100 \mathrm{mV}$ $s^{-1}$ the cell show a characteristic resistivity shape due to increase in resistance which could be ascribed to the capacitance decrease observed in Figure 5 (c). The specific capacitance calculated from the $\mathrm{CV}$ using equation 1 ranges from $74 \mathrm{~F} \mathrm{~g}^{-1}\left(5 \mathrm{mV} \mathrm{s}^{-1}\right)$ to $58 \mathrm{~F} \mathrm{~g}^{-1}\left(100 \mathrm{mV} \mathrm{s}^{-}\right.$ $\left.{ }^{1}\right)$. The constant current charge discharge curve is shown in Figure 5 (b) exhibiting a linear voltage-time function with symmetric triangular shape confirming the formation of a double layer capacitance behavior and also suggesting reversible adsorption/desorption of ions on the surface of the polarized electrode. The specific capacitance $C_{s p}$ of the cell was calculated from equations 1 and 2. The values were $58 \mathrm{~F} \mathrm{~g}^{-1}\left(10.8 \mathrm{~F} \mathrm{~cm}^{-3}\right)$ and $65 \mathrm{~F} \mathrm{~g}^{-1}\left(12.1 \mathrm{~F} \mathrm{~cm}^{-3}\right)$ at scan rate of $100 \mathrm{mVs}^{-1}$ and current density of $0.2 \mathrm{Ag}^{-1}$ respectively, where the volumetric capacitance was obtained by multiplying the gravimetric capacitance with density value of $0.1864 \mathrm{~g} \mathrm{~cm}^{-3}$ obtained from the pycnometer. These values in agreement with those obtained from the CV measurements.

Figure 5 (c) shows the Ragone plot and specific capacitance of the tested device. The capacitance of the cell reaches $65 \mathrm{Fg}^{-1}\left(12.1 \mathrm{~F} \mathrm{~cm}^{-3}\right)$ at a current density of $0.2 \mathrm{Ag}^{-1}$, exhibit energy density up to $12 \mathrm{Wh} \mathrm{kg}^{-1}$ and can deliver a power density of $0.4 \mathrm{~kW} \mathrm{~kg}^{-1}$, which are even higher than those reported in which AC//AC electrode were used $[32,33]$ and can also be compared to symmetric supercapacitor based on oxygen-enriched activated carbons from pomelo peel which had a voltage window of $1.7 \mathrm{~V}$, a specific capacitance of $43.5 \mathrm{~F} \mathrm{~g}^{-1}$ and energy density of $17.1 \mathrm{Wh} \mathrm{kg}^{-1}$ [34]. The energy and power densities values of the AGF 

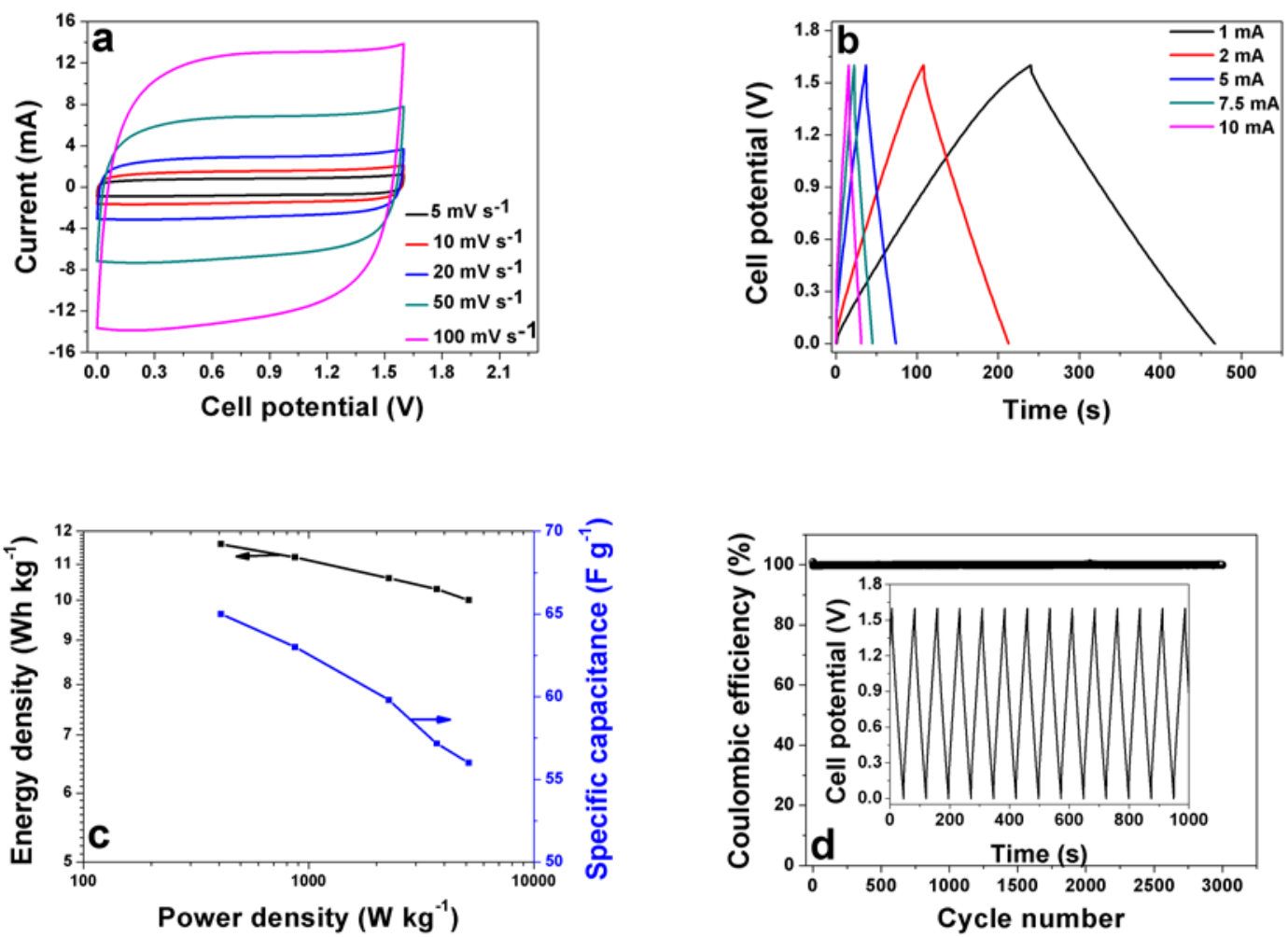

Figure 5 The CVs of the symmetric AGF supercapacitor cell operated between 0 and $1.6 \mathrm{~V}$ at different rates from 5 to $100 \mathrm{mV} \mathrm{s}-1$, (b) The galvanostatic charge-discharge curves of the symmetric supercapacitor at different currents, (c) The specific capacitance of the symmetric supercapacitor and the Ragone plot of the cell and (d) The cycle performance of the symmetric supercapacitor in the voltage window between 0 and $1.6 \mathrm{~V}$ at a current of $5 \mathrm{~mA}$. 
electrode could be attributed to the excellent materials structural properties such as high specific surface area, suitable pore size distribution and partially ordered graphitic structure [35]. The device was subjected to a constant current charge-discharge for about 3000 cycles at a current density of $1 \mathrm{~A} \mathrm{~g}^{-1}$ to test for cycle lifetime of the porous carbon electrode, and the cell retained $100 \%$ of its initial capacitance (Figure $5 \mathrm{~d}$ ), possibly due to the slow long charging and discharging which might help the ions in the electrolyte to fully access the AGF electrodes to take full advantages of the surface area by intercalating into the spaces between the sample cavities and therefore, producing more surface area for the ions to access [36]. This is an indication that the produced electrode material can be operated for practical application. Although such number of cycles may be insufficient from a practical, industrial and commercial point of view, it is useful and necessary in anticipating the lifetime and stability of the cell.

Finally, the electrochemical impedance spectroscopy (EIS) in Figure 6 (a) shows a fast ion transport mechanism of the AGF porous electrodes. A complex plane plot of the impedance (Nyquist diagram) shown in the figure with an expanded view in the inset is provided. The EIS spectrum has a semi-circular arc in the high-frequency region and a straight line in the low-frequency region, indicating a capacitive behavior. In the high-frequency region, the intercept point value in the real axis of the Nyquist plots represents the ohmic resistance of the electrode material and electrolytes $\left(R_{s}\right.$ or ESR) and the electronic resistance within the electrode material estimated from the Nyquist plot was found to be $\sim 5$ ohms. At the midfrequency region a semi-circular arc exist and the diameter of the semi-circular arc is a measure of the interfacial charge transfer resistance $\left(R_{c t}\right)$, which is related to the interface between the electrode and electrolyte, and the electrical charge transfer in Faradic process 

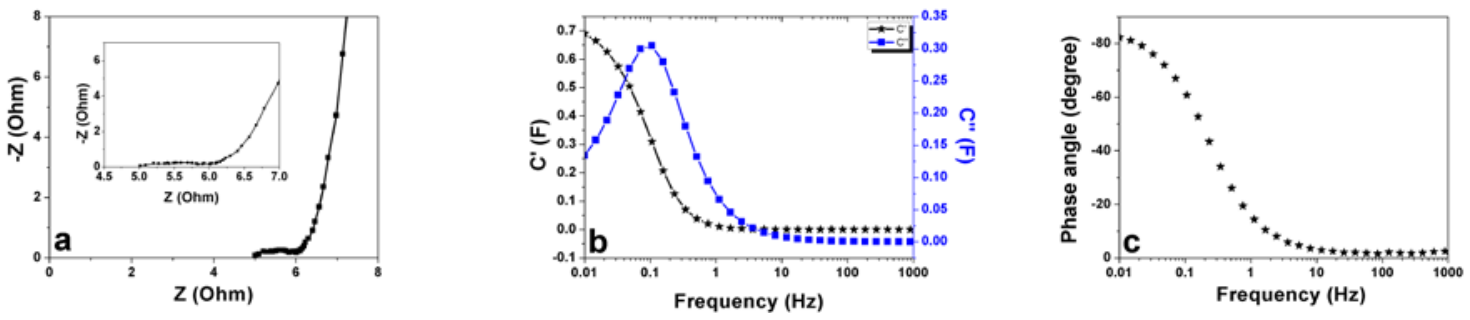

Figure 6 EIS characteristics of the cell: (a) Nyquist plot with inset showing the semicircle, (b) the real part of the cells capacitance against frequency, (c) the phase angle versus frequency of the cell. 
of the electrode [37]. The $R_{c t}$ value from the graph was approximated to be 6 Ohms; this high value could be due to long-range diffusion and transport pathways in the electrode material. The vertical line in the low frequency region is a result of the frequency dependence of ion diffusion at the electrolyte/electrode interface. The nearly vertical line at this region is a good indication of a pure capacitive behaviour with low diffusion resistance of ions in the structure of the electrode due to the mesoporous surface network, which provides better accessibility to the ions in the electrolyte. At the low frequency region, it is also observed that the vertical is inclined to the real axis suggesting a deviation from the ideal behavior. This deviation could be attributed to leakage resistance [37].

The frequency response of porous carbon electrodes has been modelled with a single series resistor-capacitor $(R C)$ circuit [38]. Based on this model, the real and imaginary part of the capacitance as a function of the frequency can be calculated using equations below.

$C=-1 /\left(\omega Z^{\prime \prime}\right)$

$C(\omega)=C^{\prime}(\omega)-j C^{\prime \prime}(\omega)$

$C^{\prime}(\omega)=\frac{-Z^{\prime \prime}(\omega)}{\omega|Z(\omega)|^{2}}$

$C^{\prime \prime}(\omega)=\frac{Z^{\prime}(\omega)}{\omega|Z(\omega)|^{2}}$

where $Z$ is the complex impedance written as $Z(\omega)=Z^{\prime}(\omega)+j Z^{\prime \prime}(\omega), \omega=2 \pi f, Z^{\prime}$ and $Z^{\prime \prime}$ represents the real and imaginary parts of the Nyquist plot respectively. $C^{\prime}(\omega)$ represents the real accessible capacitance of the cell at the corresponding frequency which represents the deliverable capacitance, while $C^{\prime \prime}(\omega)$ represents the energy loss due to the irreversible 
process of the electrodes [38]. Based on this, the real capacitances has been calculated to be $0.7 \mathrm{~F}$ (intercept on the vertical axis of Figure 6 (b) ) which represent the deliverable capacitance as a function of frequency and imaginary capacitance was used to estimate the time constant. The characteristic frequency $\left(f_{0}\right)$ which corresponds to peak of imaginary capacitance is about $0.23 \mathrm{~Hz}$ and this frequency represent the point where the capacitive and resistive impedances are the same [38]. The corresponding time constant $\tau=1 / f_{0}=4 \mathrm{~s}$ is a measure of how fast the cell could be fully charged and discharged reversibly. This rapid frequency response of $4 \mathrm{~s}$ could be attributed to the fairly large surface area with a relative amount of mesopores which enhances the ion transport rate of the device [38]. The dependency of the phase angle on the frequency is shown in Figure 6 (c). The phase angle is about $-82^{\circ}$ (ideal situation should be $90^{\circ}$ ) which suggest that the cell functionality is close to that of an ideal capacitor.

\section{$4 \quad$ Conclusions}

In summary, highly porous polarizable three dimensional activated carbon material based on graphene foam (AGF) was investigated electrodes for symmetric supercapacitor in aqueous electrolyte operating at a stable voltage of $1.6 \mathrm{~V}$. The AGF exhibited a specific

capacitance, $65 \mathrm{~F} \mathrm{~g}^{-1}$ in aqueous electrolyte, energy density of $12 \mathrm{Wh} \mathrm{kg}^{-1}$, power density of $0.4 \mathrm{~kW} \mathrm{~kg}^{-1}$ and good rate capability. These values in aqueous electrolytic media are ascribed to the excellent structural properties of the AGF which provides easy accessibility to the ions within the electrolyte and hence an increase in the electrode performance of the symmetric supercapacitor cell. Such promising results of AGF represent the potential and practical applicability of the material for low cost and high performance supercapacitor devices. 


\section{Acknowledgement}

This work is based upon research supported by the South African Research Chairs Initiative of the South African Department of Science and Technology (SARCHi-DST) and the National Research Foundation (NRF). Any opinion, findings and conclusions or recommendations expressed in this work are those of authors and therefore the NRF and DST do not accept any liability with regard thereto. A. Bello acknowledges University of Pretoria and NRF financial support for his Postdoc fellowship, while D. Y. Momodu and F. Barzegar acknowledge financial support from University of Pretoria and the NRF for PhD bursaries.

\section{References}

[1] P. Simon, Y. Gogotsi, Capacitive energy storage in nanostructured carbon--electrolyte systems, Acc. Chem. Res. 46 (2012) 1094-1103.

[2] B.E. Conway, Electrochemical supercapacitors, 1999.

[3] P. Simon, Y. Gogotsi, Materials for electrochemical capacitors, Nat. Mater. 7 (2008) 845-854.

[4] P. Hapiot, C. Lagrost, Electrochemical reactivity in room-temperature ionic liquids, Chem. Rev. 108 (2008) 2238-2264.

[5] H. Xia, Y. Shirley Meng, G. Yuan, C. Cui, L. Lu, Y.S. Meng, A symmetric $\mathrm{RuO}_{2} / \mathrm{RuO}_{2}$ supercapacitor operating at $1.6 \mathrm{~V}$ by using a neutral aqueous electrolyte, Electrochem. Solid-State Lett. 15 (2012) A60-A63.

[6] J.W. Long, D. Bélanger, T. Brousse, W. Sugimoto, M.B. Sassin, O. Crosnier, Asymmetric electrochemical capacitors-stretching the limits of aqueous electrolytes, Mrs Bull. 36 (2011) 513-522.

[7] M. Inagaki, H. Konno, O. Tanaike, Carbon materials for electrochemical capacitors, J. Power Sources. 195 (2010) 7880-7903.

[8] T.A. Centeno, M. Sevilla, A.B. Fuertes, F. Stoeckli, On the electrical double-layer capacitance of mesoporous templated carbons, Carbon 43 (2005) 3012-3015. 
[9] M. Zhi, C. Xiang, J. Li, M. Li, N. Wu, Nanostructured carbon--metal oxide composite electrodes for supercapacitors: a review, Nanoscale. 5 (2013) 72-88.

[10] M. Zhi, F. Yang, F. Meng, M. Li, A. Manivannan, N. Wu, Effects of Pore Structure on Performance of An Activated-Carbon Supercapacitor Electrode Recycled from Scrap Waste Tire, ACS Sustain. Chem. Eng. (2014).

[11] T. Kyotani, T. Nagai, S. Inoue, A. Tomita, Formation of new type of porous carbon by carbonization in zeolite nanochannels, Chem. Mater. 9 (1997) 609-615.

[12] A. Ahmadpour, D.D. Do, The preparation of active carbons from coal by chemical and physical activation, Carbon 34 (1996) 471-479.

[13] X. Gui, J. Wei, K. Wang, A. Cao, H. Zhu, Y. Jia, et al., Carbon nanotube sponges, Adv. Mater. 22 (2010) 617-621.

[14] H. Nishihara, T. Kyotani, Templated nanocarbons for energy storage, Adv. Mater. 24 (2012) 4473-4498.

[15] C. Yu, J. Fan, B. Tian, D. Zhao, G.D. Stucky, High-Yield Synthesis of Periodic Mesoporous Silica Rods and Their Replication to Mesoporous Carbon Rods, Adv. Mater. 14 (2002) 1742-1745.

[16] T. Kyotani, Z. Ma, A. Tomita, Template synthesis of novel porous carbons using various types of zeolites, Carbon N 41 (2003) 1451-1459.

[17] B. Basavalingu, J.M. Calderon Moreno, K. Byrappa, Y.G. Gogotsi, M. Yoshimura, Decomposition of silicon carbide in the presence of organic compounds under hydrothermal conditions, Carbon 39 (2001) 1763-1766.

[18] M.A. Lillo-Ródenas, D. Cazorla-Amorós, A. Linares-Solano, Understanding chemical reactions between carbons and $\mathrm{NaOH}$ and $\mathrm{KOH}$ : an insight into the chemical activation mechanism, Carbon 41 (2003) 267-275.

[19] E. Raymundo-Pinero, P. Azais, T. Cacciaguerra, D. Cazorla-Amorós, A. Linares-Solano, F. Béguin, $\mathrm{KOH}$ and $\mathrm{NaOH}$ activation mechanisms of multiwalled carbon nanotubes with different structural organisation, Carbon 43 (2005) 786-795.

[20] T. Wang, S. Tan, C. Liang, Preparation and characterization of activated carbon from wood via microwave-induced $\mathrm{ZnCl}_{2}$ activation, Carbon N 47 (2009) 1880-1883.

[21] Q.-S. Liu, T. Zheng, P. Wang, L. Guo, Preparation and characterization of activated carbon from bamboo by microwave-induced phosphoric acid activation, Ind. Crops Prod. 31 (2010) 233-238.

[22] J. Wang, S. Kaskel, $\mathrm{KOH}$ activation of carbon-based materials for energy storage, J. Mater. Chem. 22 (2012) 23710-23725. 
[23] S. Murali, N. Quarles, L.L. Zhang, J.R. Potts, Z. Tan, Y. Lu, et al., Volumetric capacitance of compressed activated microwave-expanded graphite oxide (a-MEGO) electrodes, Nano Energy. 2 (2013) 764-768.

[24] Y. Zhu, S. Murali, M.D. Stoller, K.J. Ganesh, W. Cai, P.J. Ferreira, et al., Carbon-based supercapacitors produced by activation of graphene, Science 332 (2011) 1537-1541.

[25] L. Zhang, F. Zhang, X. Yang, G. Long, Y. Wu, T. Zhang, et al., Porous 3D graphenebased bulk materials with exceptional high surface area and excellent conductivity for supercapacitors, Sci. Rep. 3 (2013).

[26] A. Bello, O.O. Fashedemi, J.N. Lekitima, M. Fabiane, D. Dodoo-Arhin, K.I. Ozoemena, et al., High-performance symmetric electrochemical capacitor based on graphene foam and nanostructured manganese oxide, AIP Adv. 3 (2013) 82118.

[27] M.D. Stoller, R.S. Ruoff, Best practice methods for determining an electrode material's performance for ultracapacitors, Energy Environ. Sci. 3 (2010) 1294-1301.

[28] Y. Gogotsi, P. Simon, Materials science. True performance metrics in electrochemical energy storage., Science 334 (2011) 917-8.

[29] W. Gao, L.B. Alemany, L. Ci, P.M. Ajayan, New insights into the structure and reduction of graphite oxide, Nat. Chem. 1 (2009) 403-408.

[30] K.S.W. Sing, Reporting physisorption data for gas/solid systems with special reference to the determination of surface area and porosity, Pure Appl. Chem. 57 (1985) 603619.

[31] C.G. V Burgess, D.H. Everett, S. Nuttall, Adsorption hysteresis in porous materials, Pure Appl. Chem. 61 (1989) 1845-1852.

[32] L. Demarconnay, E. Raymundo-Pinero, F. Béguin, A symmetric carbon/carbon supercapacitor operating at $1.6 \mathrm{~V}$ by using a neutral aqueous solution, Electrochem. Commun. 12 (2010) 1275-1278.

[33] B. Xu, F. Wu, R. Chen, G. Cao, S. Chen, G. Wang, et al., Room temperature molten salt as electrolyte for carbon nanotube-based electric double layer capacitors, J. Power Sources 158 (2006) 773-778.

[34] C. Peng, J. Lang, S. Xu, X. Wang, Oxygen-enriched activated carbons from pomelo peel in high energy density supercapacitors, RSC Adv. 4 (2014) 54662-54667.

[35] C. Zhang, K.B. Hatzell, M. Boota, B. Dyatkin, M. Beidaghi, D. Long, et al., Highly porous carbon spheres for electrochemical capacitors and capacitive flowable suspension electrodes, Carbon (2014).

[36] Q. Cheng, J. Tang, J. Ma, H. Zhang, N. Shinya, L.-C. Qin, Graphene and nanostructured MnO2 composite electrodes for supercapacitors, Carbon 49 (2011) 2917-2925. 
[37] X. Zhang, X. Wang, L. Jiang, H. Wu, C. Wu, J. Su, Effect of aqueous electrolytes on the electrochemical behaviors of supercapacitors based on hierarchically porous carbons, J. Power Sources. 216 (2012) 290-296.

[38] P.L. Taberna, P. Simon, J.-F. Fauvarque, Electrochemical characteristics and impedance spectroscopy studies of carbon-carbon supercapacitors, J. Electrochem. Soc. 150 (2003) A292-A300. 